\title{
MACROFAUNA PRESENTE NAS MATRIZES DE Byrsonima gardneriana A. Juss NA CAATINGA DE OLHO D'ÁGUA DO CASADO, ALAGOAS
}

\author{
Delane dos Santos Dias ${ }^{1}$, Ana Beatriz da Silva², Elba dos Santos Lira ${ }^{3}$, Nivaneide Alves de Melo4, Kallianna \\ Dantas Araujo ${ }^{5}$ \\ Parte do trabalho de PIBIC (Ciclo 2017-2018) \\ 'Discente do Curso de Geografia (Licenciatura)-Instituto de Geografia, Desenvolvimento e Meio Ambiente-IGDEMA, Universidade \\ Federal de Alagoas, CEP: 57072-970, Maceió-Alagoas, E-mail: delane.sd@hotmail.com \\ 2Discente do PPGG/IGDEMA/UFAL, E-mail: anabtrizsilva@gmail.com \\ ${ }^{3}$ Mestra em Geografia pelo PPGG/IGDEMA/UFAL, E-mail: elbaslira@yahoo.com.br \\ 4,5Docentes do PPGG/IGDEMA/UFAL, E-mail: nivaneide.ufal@yahoo.com.br; kallianna.araujo@igdema.ufal.br
}

\begin{abstract}
RESUMO: A macrofauna é constituída por organismos com comprimento $\geq 2,0 \mathrm{~mm}$, importante no sucesso reprodutivo das plantas por atuarem como agentes polinizadores, sendo relevante o conhecimento da interação desses organismos com espécies vegetais, notadamente $B$. gardneriana A. Juss (Murici), pela já utilizada como fonte de alimento para a fauna silvestre, animais domésticos e população. Objetivou-se avaliar a abundância, riqueza, diversidade e equabilidade da macrofauna em área com presença da espécie $B$. gardneriana $A$. Juss na Caatinga de Olho D'Água do Casado, Alagoas. A macrofauna foi quantificada, nos períodos (set. e dez./2017) e (mar. e jun./2018), por meio de armadilhas Provid aérea em 15 matrizes da espécie B. gardneriana. Os organismos foram identificados a nível de grupo taxonômico e foi avaliado a riqueza, abundância, diversidade e uniformidade pelos Índices de Shannon $(H)$ e Pielou $(e)$. Foram coletados dados de precipitação pluvial mensalmente para relacionar com a macrofauna. Hymenoptera é o grupo dominante no ambiente com ocorrência de $B$. gardneriana, independente dos períodos avaliados, comprovado pelos baixos valores dos índices de Shannon $(\mathrm{H})$ e Pielou (e); A abundância dos organismos é maior em dez/2017, atribuída ao grupo Hymenoptera, já adaptado a variabilidade das condições microclimáticas da área.
\end{abstract}

Palavras-chave: Semiárido, Organismos invertebrados, Murici.

\section{MACROFAUNA PRESENT IN THE Byrsonima gardneriana A. Juss matrices IN CAATINGA DE OLHO D'ÁGUA DO CASADO, ALAGOAS}

\begin{abstract}
Macrofauna consists of organisms with a length $\geq 2.0 \mathrm{~mm}$, which is important in reproductive success of plants by acting as pollinators' agents, being relevant knowledge of these organisms interaction with plant species, notably B. gardneriana A. Juss (Murici), already used as food source for wildlife, domestic animals and population. The aim of this study was to evaluate the abundance, richness, diversity and equability of macrofauna in an area with presence of B. gardneriana A. Juss in Caatinga, Olho D'Água do Casado, Alagoas. Macrofauna were quantified, in periods (set and dec/2017) and (mar and jun/2018), by aerial Provid traps in 15 matrices of $B$. gardneriana. Organisms were identified at the level of taxonomic group and it was evaluated richness, abundance, diversity and uniformity by the Shannon $(\mathrm{H})$ and Pielou $(\mathrm{e})$ indices. It was collected rainfall data monthly to relate to the macrofauna. Hymenoptera is the dominant group in the environment with $B$. gardneriana occurrence, independent of the evaluated periods, as evidenced by the low values of the Shannon $(\mathrm{H})$ and Pielou $(\mathrm{e})$ indices; the abundance of organisms is greatest in Dec / 2017, attributed to the Hymenoptera group, already adapted to the variability of the microclimatic conditions of the area.
\end{abstract}

Keywords: Semiarid, Invertebrate organisms, Murici. 


\section{INTRODUÇÃO}

O sucesso reprodutivo das plantas ocorre em virtude da relação que mantêm com os organismos invertebrados, já que estes atuam na ciclagem de nutrientes, preparando os minerais que são levados para as plantas através das raízes, e na copa da árvore atuam como polinizadores e dispersores de sementes (LEAL, 2012).

Dentre as espécies da Caatinga Alagoana, destaca-se Byrsonima gardneriana A. Juss adaptada as condições do ambiente, fonte de alimento para a fauna silvestre, animais domésticos e população local (SOUZA, 2011), sendo necessário estudos sobre a relação da macrofauna aérea com esta espécie.

A macrofauna corresponde aos organismos com comprimento $\geq 2,0 \mathrm{~mm}$, destacando-se Hymenoptera, Coleoptera, Orthoptera, dentre outros (GIRACCA et al., 2003).

0 objetivo do trabalho foi avaliar a abundância, riqueza, diversidade e equabilidade da macrofauna em área com presença da espécie Byrsonima gardneriana A. Juss na Caatinga de Olho D’Água do Casado, Alagoas.

\section{MATERIAL E MÉTODOS}

A pesquisa foi realizada em Olho D'Água do Casado, coordenadas geográficas $10^{\circ} 03^{\prime} 30^{\prime \prime}$ $S$ e $36^{\circ} 49^{\prime} 00^{\prime \prime} \mathrm{W}$, altitude de $230 \mathrm{~m}$, inseridos na Mesorregião Geográfica do Sertão e Microrregião Geográfica Alagoana do Sertão do São Francisco.

O clima da área de estudo é Tropical Semiárido (BSh), segundo a classificação de Köppen (PEEL et al., 2007). Os solos predominantes são Neossolos Litólicos, constituídos por fragmentos pedregosos e Neossolos Flúvicos, derivados de ambientes com sedimentação aluvial (EMBRAPA, 2013) e a vegetação característica é a Caatinga Hipoxerófila, com trechos de Floresta Caducifólia (SOUZA, 2014).

$A$ determinação da macrofauna aérea foi realizada nas 15 matrizes da espécie $B$. gardneriana nos meses setembro e dezembro/2017, março e junho/2018. Na avaliação da macrofauna foi utilizada armadilhas Provid (ARAUJO, 2010), constituídas de garrafa PET $2 \mathrm{~L}$, contendo $200 \mathrm{~mL}$ de solução de detergente, na concentração de $5 \%$ e 12 gotas de Formol P.A. (Formaldeído) e permaneceram no campo por 96 horas. As armadilhas foram instaladas na copa das árvores de $B$. gardneriana na altura de $1,50 \mathrm{~m}$ do solo.

Foi avaliada a diversidade pelo Índice de Shannon $(H)$ definido por: $H=-\Sigma$ pi.logpi, em que: $\mathrm{pi}=\mathrm{ni} / \mathrm{N} ; \mathrm{ni}=$ densidade de cada grupo e a equabilidade pelo Índice de Pielou (e) definido por: $e=H / \log S$, em que: $\mathrm{H}=$ =índice de Shannon; $\mathrm{S}=$ Número de espécies ou grupos. 0 índice $(\mathrm{H})$ varia de 0 a 5 , indicando que 0 declínio de seus valores é o resultado de uma maior dominância de grupos em detrimento de outros.

Foi feita a identificação dos organismos com lupa binocular e chave de identificação de Triplehorn e Johnson (2011), no Laboratório de Ecogeografia e Sustentabilidade Ambiental-LABESA/IGDEMA/UFAL.

Foram realizadas medidas mensais de precipitação pluvial com pluviômetro Ville de Paris instalado próximo a área pesquisada.

\section{RESULTADOS E DISCUSSÃO}

Foram capturados 263 organismos na parte aérea das matrizes de Byrsonima gardneriana $A$. Juss (Murici) (Gráfico 1A), distribuídos em 10 grupos taxonômicos (Gráficos 1B).

$\mathrm{Na}$ avaliação feita em dez/2017, não houve registro de precipitação pluvial (Gráfico 1B), influenciando a baixa riqueza, predominando os grupos resistentes às condições microclimáticas locais mais extremas, como Hymenoptera, que apresentou elevado número de indivíduos $(89,42 \%)$, seguido de Araneae (3,85\%) (Gráfico 2B).

Gráfico 1. Abundância $(A)$ e riqueza $(B)$ da macrofauna aérea relacionada com precipitação pluvial $(\mathrm{mm})$, em Olho D'Água do Casado, Alagoas.

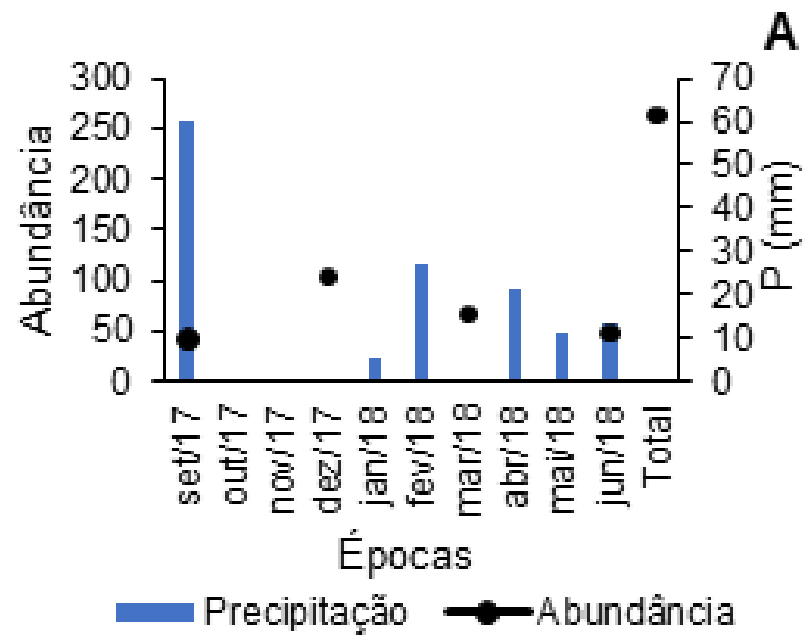




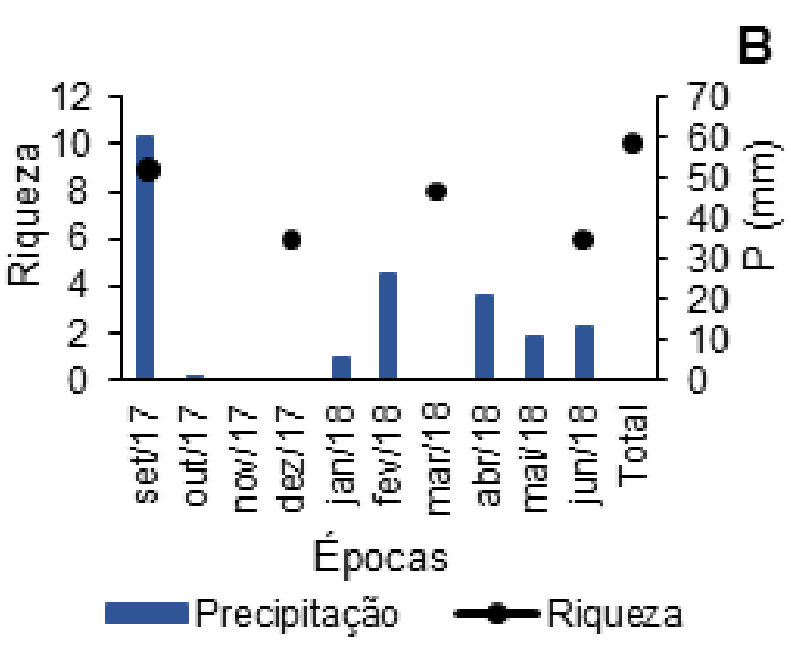

A riqueza da macrofauna aérea foi favorecida pela precipitação pluvial (Gráfico 1B). Em mar/2018, mesmo não havendo registro de precipitação pluvial, a riqueza foi elevada decorrente da precipitação pluvial registrada nos meses anteriores $($ jan $/ 2018=5,4 \mathrm{~mm}$; fev $/ 2018=$ 26,7 mm) (Gráfico 1B).

$\mathrm{Na}$ determinação realizada em jun/2018, houve registro de alta precipitação pluvial (13,3 $\mathrm{mm}$ ), possibilitando 0 aumento da riqueza, favorecida pelas condições favoráveis de precipitação pluvial (mm) (Gráfico 1B). Os grupos taxonômicos Hymenoptera $(41,67 \%)$ e Araneae $(29,17 \%)$ foram os mais abundantes registrados (Gráfico 2D).

Gráfico 2. Percentual da macrofauna aérea capturados em set/2017 (A), dez/2017 (B), mar/2017 (C) e jun/2018 (D), em Olho D’Água do Casado, Alagoas.
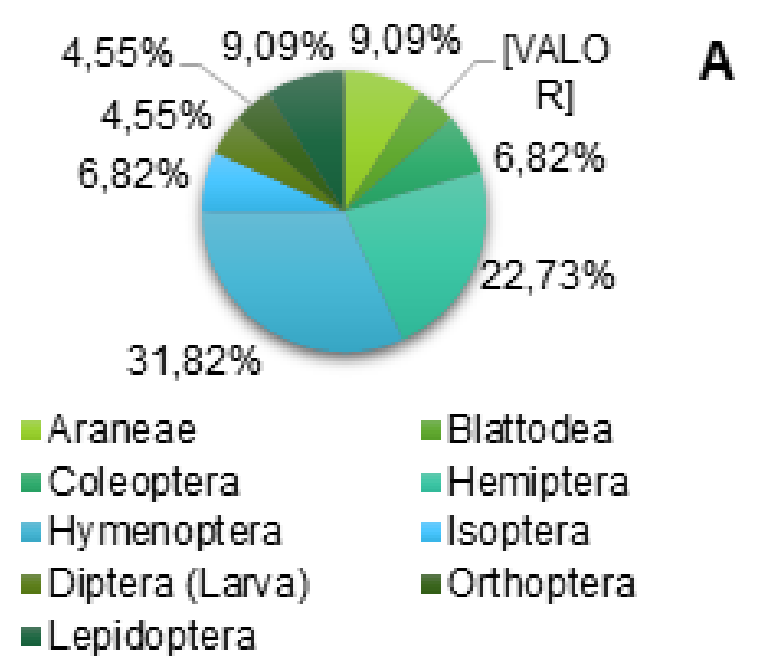
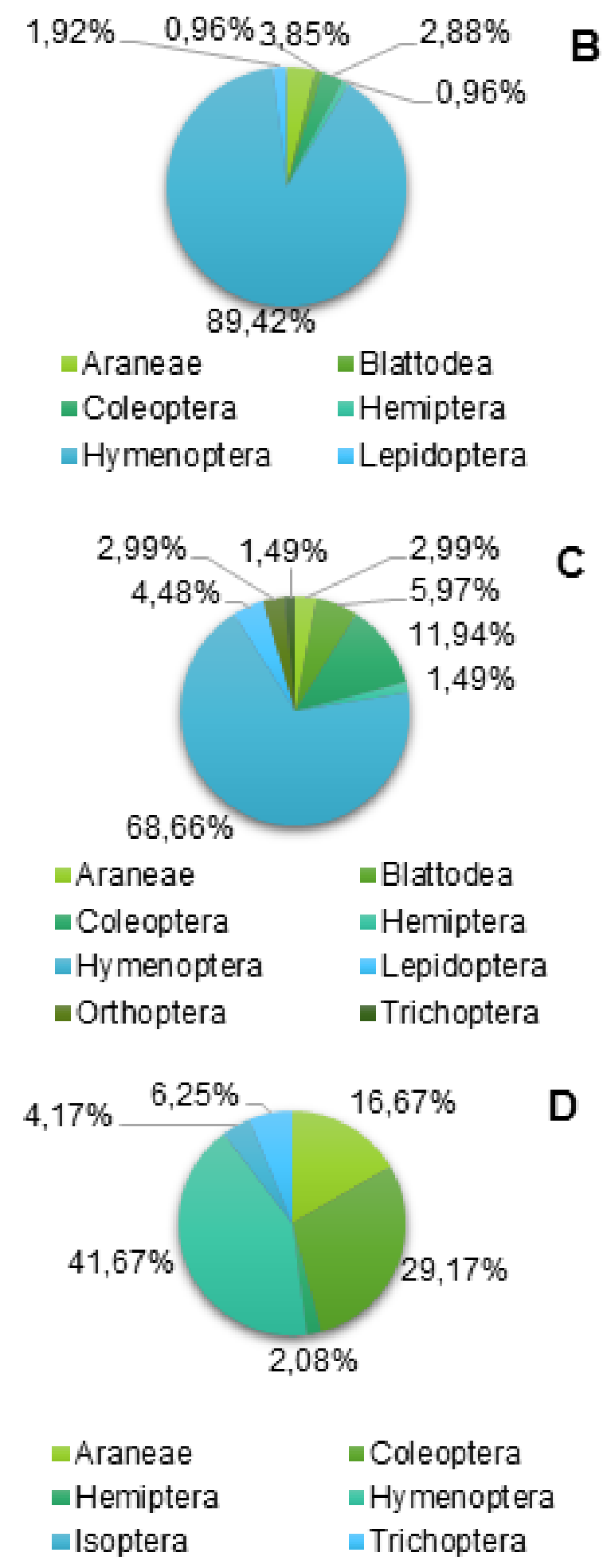

O grupo Hymenoptera foi mais abundante apresentando maior percentual, independente da época do ano (Gráficos 2A a 2D). Alves (2018) também constatou alto registro do grupo Hymenoptera, no município Olho D’Água das Flores, Alagoas, em ambiente Semiárido. Menezes et al. (2009) explicam que a dominância deste grupo, principalmente formigas, nas diferentes épocas do ano, decorre da alta adaptabilidade as variedades do meio, à alimentação e por viverem em sociedade. 
A menor diversidade e uniformidade correspondeu ao grupo Hymenoptera $(H=0,18$; $e=$ $0,80)$ e Coleoptera $(H=0,97 ; e=0,40)$ (Gráfico 3). Alves (2018), em coletas utilizando armadilhas Provid aérea instalada na copa das árvores, em ambiente de Caatinga de Olho D'Água das Flores, Alagoas, registrou a presença de Hymenoptera com os menores valores de diversidade, seguido de Coleoptera. De acordo com Begon (1996), quanto menores os valores de diversidade, mais dominante é o grupo taxonômico em relação aos outros.

Gráfico 5. Índice de diversidade de Shannon $(\mathrm{H})$ e índice de uniformidade de Pielou (e) para os grupos taxonômicos registrados.

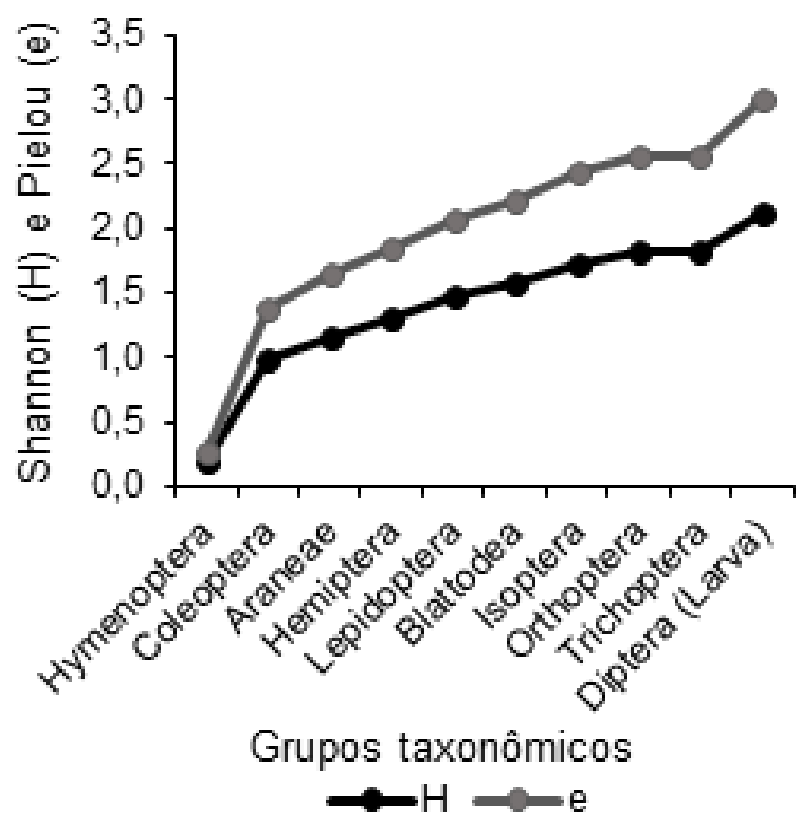

\section{CONCLUSÕES}

Hymenoptera é o grupo dominante no ambiente com ocorrência de Byrsonima gardneriana A. Juss, independente dos períodos avaliados, comprovado pelos baixos valores dos índices de Shannon $(\mathrm{H})$ e Pielou (e); A abundância dos organismos é maior em dez/2017, atribuída ao grupo Hymenoptera, já adaptado a variabilidade das condições microclimáticas da área.

\section{AGRADECIMENTOS}

Ao CNPq pela concessão de bolsa de estudo e ao Laboratório de Ecogeografia e Sustentabilidade Ambiental-IGDEMA/UFAL, pela identificação dos grupos taxonômicos da macrofauna aérea.

\section{REFERÊNCIAS}

ALVES, S. da S. Dinâmica da macrofauna na serapilheira em áreas com plantio de Eucalyptus ssp. e Caatinga, em Olho D’Água das Flores, Semiárido de Alagoas. 2018. 95 f. Dissertação (Mestrado em Geografia)-Instituto de Geografia, Desenvolvimento e Meio Ambiente, Universidade Federal de Alagoas, Maceió, 2018.

BEGON, M.; HARPER, J. L.; TOWNSEND, C. R. Ecology: individuals, populations and communities. 3. ed. Oxford: Blackwell Science, 1996, 1068 p.

ARAUJO, K. D. Análise da vegetação e organismos edáficos em áreas de caatinga sob pastejo e aspectos socioeconômicos e ambientes de São João do Cariri - PB. 2010. 166 f. Tese (Doutorado em Recursos Naturais)-Centro de Tecnologia e Recursos Naturais, Universidade Federal de Campina Grande, Campina Grande, 2010.

EMBRAPA-EMPRESA BRASILEIRA DE PESQUISA AGROPECUÁRIA. Zoneamento agroecológico de Alagoas: levantamento de reconhecimento de baixa e média intensidade dos solos do Estado de Alagoas. 1. ed. Recife: EMBRAPA, 2013. 238 p.

GIRACCA, E. M. N. et al. Levantamento da meso e macrofauna do solo na microbacia de Arroio Lino, Agudo/RS. Revista Brasileira de Agrociência, 2003, 9, 257-261.

LEAL, I. R. Diversidade de formigas em diferentes unidades de paisagem da Caatinga. In: LEAL, I. R.; TABARELLI, M.; SILVA, J. M. C. da. Ecologia Brasileira de Biociência, 2012, 10, 446-456.

PEEL, M. C.; FINLAYSON, B. L.; MCMAHON, T. A. Updated world map of the Köppen-Geiger climate classification. Hydrology and Earth System Sciences, 2007, 5, 1633-1644.

SOUZA, M. A. Dinâmica da serapilheira e fauna edáfica em áreas de murici (Byrsonima gardneriana 
A. Juss) no Semiárido de Alagoas, Brasil. 2014. 138 f. Tese (Doutorado em Agronomia)-Centro de Ciências Agrárias, Universidade Federal da Paraíba, Areia, 2014.

SOUZA, M. A. Fitossociologia em áreas de Caatinga e conhecimento etnobotânico do Murici (Byrsonima gardneriana A. Juss), Semiárido Alagoano. 2011. 88 f. Dissertação (Mestrado em Agronomia)-Centro de Ciências Agrárias, Universidade Federal da Paraíba, Areia, 2011.
TRIPLEHORN, C. A.; JONNSON, N. F. Estudo dos insetos. 7. ed. São Paulo: Cengage Learning, 2011. $809 \mathrm{p}$. 